\title{
Assessing personality across 13 countries using the California Adult Q-set
}

\author{
Gwendolyn Gardiner ${ }^{1}$, Esther Guillaume ${ }^{2}$, Nick Stauner ${ }^{3}$, Jaechang Bae ${ }^{4}$, Gyuseong Han ${ }^{4}$, \\ Jungsoon Moon ${ }^{4}$, Igor Bronin ${ }^{5}$, Christina Ivanova ${ }^{5}$, Joey T. Cheng ${ }^{6}$, François De Kock ${ }^{7}$, \\ Sylvie $\mathrm{Graf}^{8}$, Martina Hřebičcková ${ }^{8}$, Peter Halama ${ }^{9}$, Ryan Hong ${ }^{10}$, Paweł Izdebski ${ }^{11}$, \\ Clara Kulich ${ }^{12}$, Fabio Lorenzi-Cioldi ${ }^{12}$, Lars Penke ${ }^{13}$, Piotr Szarota ${ }^{14}$, \\ Jessica Tracy ${ }^{15}$, Yu Yang ${ }^{16}$, David Funder ${ }^{1}$ \\ ${ }^{1}$ University of California, Riverside \\ ${ }^{2}$ Chapman University \\ ${ }^{3}$ Case Western Reserve University \\ ${ }^{4}$ Chonnam National University \\ ${ }^{5}$ Ural Federal University \\ ${ }^{6}$ University of Illinois, Urbana-Champaign \\ ${ }^{7}$ University of Cape Town \\ ${ }^{8}$ Academy of Sciences of the Czech Republic \\ ${ }^{9}$ Slovak Academy of Sciences \\ ${ }^{10}$ National University of Singapore \\ ${ }^{11}$ Kazimierz Wielki University \\ ${ }^{12}$ University of Geneva \\ ${ }^{13}$ University of Goettingen \\ ${ }^{14}$ Institute of Psychology, Polish Academy of Sciences \\ ${ }^{15}$ University of British Columbia \\ ${ }^{16}$ ShanghaiTech University
}

\begin{abstract}
The current project measures personality across cultures, for the first time using a forced-choice (or idiographic) assessment instrument - the California Adult $Q$-set (CAQ). Correlations among the average personality profiles across 13 countries (total $N=2,370$ ) ranged from $r=.69$ to $r=.98$. The most similar averaged personality profiles were between USA/Canada; the least similar were South Korea/Russia/Poland and China/Russia. The Czech Republic had the most homogeneous personality descriptions and South Korea had the least. In further analyses, country differences in CAQ-derived Big Five scores were compared to results obtained from previous research using nomothetic Likert scales (i.e., the NEO; the BFI). The Big Five templates produced generally similar findings to previous research comparing the Big Five across countries using Likert-type methods.
\end{abstract}

Keywords: personality, cross-cultural personality, personality traits, cross-cultural assessment

Cross-cultural assessments of personality have revealed meaningful variation in traits around the world. By far, the most widely studied personality attributes in these assessments are the Big Five (i.e., extraversion, neuroticism, agreeableness, conscientiousness, and openness/intellect (Digman, 1990; Goldberg, 1992), which have been validated in many countries and languages (McCrae \& Costa, 1997). However, these cross-cultural assessments of the Big Five have employed nomothetic, Likert-type scales, which are susceptible to response biases that can raise problems for cross-cultural comparisons. In the present paper, we present a different method of personality assessment across countries, using an idiographic, forced-choice measurement tool that may help alleviate some response biases and allow for more meaningful comparisons.

\section{Cross-cultural assessments of personality traits}

McCrae (2001) was one of the first researchers to compare the Five Factor Model across countries, using the Revised NEO Personality Inventory (NEO-PI-R; Costa \& McCrae, 1992) in 26 countries. The Five Factor Model was replicated across countries, implying that these characteristics might be internationally applicable. Using the same data set, Costa, Terracciano, and McCrae (2001) examined gender differences across the 26 countries, and a clear pattern emerged: on average, men scored higher than women in assertiveness (a facet of extraversion) and openness to ideas (a facet of openness to experience), and women reported higher levels of neuroticism, agreeableness, warmth (a facet of agreeableness), and openness to feelings (another facet of openness to experience). Gender differences on traits, particularly neuroticism, varied across countries, with traditionally collectivist countries (e.g., African and Asian) showing smaller differences between men and women, and 
individualistic countries (e.g., European and American) displaying greater differences (Costa et al., 2001). McCrae et al. (2005) also collected informant reports of personality data from 51 countries to circumvent bias in self-reports. Average country scores and gender differences were comparable to the original findings in McCrae (2001).

Schmitt et al. (2007) extended the previous work by assessing personality traits across 56 nations using a different measure: the Big Five Inventory (BFI). Results were consistent with Allik and McCrae (2004). South American and European countries tended to be highest in openness, while Asian countries were lowest, and African countries tended to be lowest in neuroticism. The correlations between the BFI country scores from Schmitt et al. (2007) and the NEOPI-R country scores from McCrae (2002) were moderate to strong for all Big Five traits, implying good cross-instrument validity between the BFI and the NEO. Schmitt, Realo, Voracek, and Allik (2008) also examined gender differences with the BFI. Similar to Costa et al. (2001), African and Asian countries tended to have smaller gender differences in personality traits than Europe, North and South America. These cultural differences were found to be largely associated with a country's economic development, with greater gender differences in personality found in countries with higher economic development. Overall, the pattern of country trait scores derived from personality questionnaires using Likert-type scales were moderately consistent across multiple studies.

\section{Issues with cross-cultural assessments of personality traits}

When comparing personality traits across groups, it is important that the items included in measurement instruments are understood by participants in the different groups as equivalently as possible. Cross-cultural comparisons can be especially problematic because many cultural factors can influence how individuals interpret and respond to survey questions. For example, when deciding how characteristic an item is of oneself, people may compare themselves with others around them, a tendency known as the reference group effect (Heine, Lehman, Peng, \& Greenholtz, 2002). Because social norms can differ depending on cultural context, personality judgements based on implicit comparisons with one's reference group may hide or exaggerate the differences between individuals from different cultures. Additionally, various response biases, such as acquiescence bias (tending to respond "yes" to items regardless of content) and extreme response style (tending to use the ends rather than middle of a response scale) can bias scores, limiting the conclusions that can be drawn from them. Response biases have been found to vary with cultural context. For example, acquiescence bias tends to be higher in more collectivistic cultures (Smith, 2004). Thus, when comparing personality trait scores between individual and collectivistic countries, it can be difficult to discern between cultural differences in actual trait levels and cultural differences in response styles to survey questions.

The California Adult Q-set (CAQ) has the potential to help to alleviate some of these issues. Introduced by Jack Block (Block, 1978; originally published 1961), the CAQ is a an idiographic, forced choice personality assessment tool that contains a list of 100 characteristics, including "is critical, skeptical, not easily impressed," "has a wide range of interests," "is a genuinely dependable and responsible person." The items of the CAQ were revised for use by nonpsychologists by Bem and Funder (1978), and further simplified and clarified for international use (and easier translation) by Guillaume and Funder (2012). The completion of a personality description with the CAQ essentially amounts to rank-ordering the 100 descriptors into 9 categories ranging from "most characteristic" to "least characteristic." Each of the 9 categories has a fixed number of items that form a normal distribution, with the fewest items at each extreme, and most of the items distributed near the middle, neutral category ${ }^{\mathrm{i}}$. The forced-choice sorting of the Q-set items is sometimes referred to as an "idiographic" method, because it focuses on the relative placement of items compared within persons, rather than their relative placement compared across persons (Block, 1978).

The forced-choice nature of this instrument might make it particularly useful for cross-cultural research, for several reasons. First, forced-choice measures such as the CAQ may help mitigate reference group effects. When making a Likert-type (e.g., rating from 1 to 5) or true-false response, participants are implicitly invited to compare their own (or the target's) level of a trait to the average they presume exists in the salient comparison group. For example, when asked to rate one's own degree of conscientiousness, it would be reasonable to compare oneself to the level of this trait in one's local context or culture (Heine et al., 2002). If that level is generally high, then a highly conscientious person might rate oneself average or even low because of this comparison. In a forced choice measure such as the Q-sort, by contrast, the most salient comparison is not with other people in the local context, but rather with the other traits or characteristics in the Q-sort. For example, a person rating one's own degree of "dependability" with the Q-sort must place this rating into the 9-category distribution relative to 99 other characteristics such as "skeptical," "dominant," and many more. While this property of the Q-sort might not completely immunize it to reference group effects, it might serve to make the reference group comparison less salient than the inter-item comparison.

Second, the forced-choice nature of the CAQ eliminates or reduces the influence of response styles that have been a traditional concern for cross-cultural research (Ross \& Mirowsky, 1984; van de Vijver \& Leung, 1997; 2011). Extremity and acquiescence response sets cannot influence CAQ scores because the distribution of responses across the 9-category range is pre-determined (see Footnote i). Social desirability bias and the halo effect, while not necessarily wholly eliminated, would be at least somewhat reduced because the highest and lowest rating categories are not large enough to contain a majority of the desirable and undesirable items. A person - or a culture - with a tendency to rate almost all positively-valenced items as highly characteristic, for example, will find this to be impossible because only 5 of the 100 items of the CAQ may be placed into this category. Despite these potential advantages, forced-choice measures such as the CAQ have not been previously em-

${ }^{\mathrm{i}}$ Across the 9 categories, the prescribed distribution for the CAQ is 5, 8 , $12,16,18,16,12,8$, and 5 . 
ployed in the study of personality across cultures. This may be due to the construction and application of the Q-sorts.

Traditionally, Q-sorts were completed using decks of 100 cards that were literally sorted into 9 categories on a table. The equivalent procedure online requires functionality that allows participants to "sort" the items by dragging them and dropping each one into the prescribed categories. Such on-line functionality has been developed only recently (including by programmers associated with our research group).

\section{Issues with cross-cultural comparisons of personality trait scores}

Issues in cross-cultural assessment of personality may also be addressed after data collection has completed through various techniques for assessing so-called "measurement invariance" (van de Vijver \& Leung, 2001). A wide range of methods for testing measurement invariance are available, such as exploratory factor analyses, confirmatory factor analyses, principal components analyses, and their various specific applications, all of which are fundamentally similar in technique but may be most suitable only under certain methodological circumstances. This has resulted in confusion about appropriate methodological steps to employ in any given study. For example, determining the validity of personality items based on how they load on various subscales can lead to difficult interpretation issues of latent traits when the factor structure is weak or items are included that do not load on any specific latent factor (Church et al., 2011). A more fundamental limitation of statistical methods for assessing "measurement invariance" is that they are all internal to the scales themselves; they are based solely on relationships among the items on the scales and the latent factors that (sometimes) emerge.

An alternative (or perhaps, complementary) approach to assessing the similarity of meaning of measures across cultures is testing for convergent validity. The reasoning is that if two measures of a construct reveal similar patterns of mean differences across cultures, or have similar patterns of correlations with independent criteria, then the instruments likely tap into the same construct (Campbell \& Fiske, 1959; Cronbach \& Meehl, 1955). First, if similar patterns of mean differences between subscales or items on a scale appear in different cultures, this finding would strongly imply that the meaning of these subscales or items is the same across these cultures, at least insofar as their meaning is captured in their relationships with each other. For example, if Trait A consistently has a higher mean score across subjects than Trait $\mathrm{B}$, then this finding implies that the relative meaning of $\mathrm{A}$ and $\mathrm{B}$ are at least somewhat similar in the different contexts in which it was assessed. More powerfully, if a large number of traits, say a 100 , were to yield similar patterns of relative mean scores across different cultures, this would be evidence that the traits, taken as a group, maintain at least some of their meaning across these cultures.

Second, patterns of relationships between traits and independent criteria, such as cultural properties, or across independent samples, such as studies done at different times using different recruitment methods, would also support the cross-cultural meaningfulness of trait scores. This type of cross-cultural validation has begun to be increasingly employed as independent personality data sets from around the world are becoming more available. Using this approach, Schmitt et al. (2007) correlated BFI country trait scores with previously reported NEO-PI-R country trait scores (McCrae, 2002). Extraversion, conscientiousness, and neuroticism all produced good convergence across the two measures, while agreeableness and openness had moderate correlations across measures. Their results provided one of the first large-scale test of country-level broad personality trait convergence across measures, both of which were based upon traditional, Likert-style responses to items. The present study extends this approach to comparisons with a forced-choice method, the Q-sort.

\section{Purpose of present study}

The current research addresses issues in cross-cultural psychology by unifying the manner of assessment, gathering similar samples via similar methods across countries, utilizing a forced-choice instrument that may circumvent some issues with Likert scales often used in cross-cultural research (Heine et al., 2002; Ross \& Mirowsky, 1984; van de Vijver \& Leung, 1997; 2011), and by comparing results using five-factor scores derived from the CAQ with previously reported findings using other measures of the five traits and their correlates across countries. Thus, the present study is largely exploratory.

The present research will pursue six substantive goals:

1. Examine the overall CAQ item endorsement collapsed across countries. Which items are most endorsed as reflective of personality, and which items are least endorsed? In other words, what is the world-wide "average personality"?

2. Assess the average similarity of personality profiles across countries. Past research demonstrates that averaged personality profiles tend to be similar around the world (Allik \& McCrae, 2004; Allik et al., 2017; McCrae et al., 2005). As mentioned above, if this finding were to hold across the profile of 100 Q-sort items, it would support the similarity of their meaning across the cultures in which they are assessed.

3. Within each country, examine how similar each individual is to every other individual. This will assess the homogeneity of individual personality profiles within countries, in order to answer the question, "Are the personalities of inhabitants of certain countries more homogenous than inhabitants of others, and if so, which ones?"

4. Examine gender and country differences in the CAQ Big Five (using a scale built from items identified by McCrae, Costa, \& Busch 1986). In previous research, gender differences for certain characteristics have been quite large within some countries and smaller in others, appearing largest in the European and US samples and smallest in Asian and African samples (Costa et al., 2001).

5. Correlate the mean scores of the CAQ Big Five with the mean country scores of the NEO-PI-R as reported by Allik et al. (2017). 
Table 1. Characteristics of samples. Total N=2,370; Women:1,607; Men:763

\begin{tabular}{|c|c|c|c|c|c|c|c|}
\hline Country & University & Language & Compensation & $N$ & Women & Men & $M_{\text {Age }}$ \\
\hline$\overline{\text { Canada }}$ & University of British Columbia & English & course credit & 316 & 236 & 80 & 20 \\
\hline China & Several universities & Chinese & $\$ 0.67$ USD pp & 398 & 213 & 185 & 23 \\
\hline Czech Republic & 7 Universities & Czech & volunteer & 266 & 204 & 62 & 29 \\
\hline France & University of Chambery; Aix en Provence & French & volunteer & 60 & 34 & 26 & 22 \\
\hline Poland & Kazimierz Wielki University & Polish & volunteer & 79 & 66 & 13 & 26 \\
\hline Russia & Ural Federal University & Russian & course credit & 114 & 79 & 35 & 19 \\
\hline Singapore & National University of Singapore & English & course credit & 148 & 92 & 56 & 22 \\
\hline Slovakia & 3 Universities & Slovak & volunteer & 58 & 52 & 6 & 24 \\
\hline South Africa & University of Cape Town & English & course credit & 181 & 117 & 64 & 23 \\
\hline South Korea & Chonnam National University & Korean & course credit & 104 & 71 & 33 & 21 \\
\hline Switzerland & University of Geneva & French & volunteer & 106 & 77 & 29 & 25 \\
\hline UK & University of Edinburgh & English & course credit & 159 & 120 & 39 & 20 \\
\hline US & University of California, Riverside & English & course credit & 381 & 246 & 135 & 20 \\
\hline
\end{tabular}

Note: Countries including samples from multiple universities: China, Slovakia, Czech Republic; $p p=$ per person

6. Correlate the mean scores of the CAQ-derived Big Five with the mean scores of the Big Five Inventory (BFI) collected from Schmitt et al. (2007). These last two analyses, (5) and (6), are tests of convergent validity, assessing how similar country-level averages on the CAQ Big Five are to other country-level mean Big Five scores derived from independent samples and assessed with instruments that employ traditional Likert scales.

\section{METHOD}

\section{Participants}

All participants ( $N=2,370$; women: 1,607 ; men: 763$)$ were members of college/university communities, primarily students, and recruited by research collaborators in 13 countries, on 5 continents, using 9 languages. Table 1 displays characteristics of each of the samples.

\section{Measure}

As was described earlier, the California Adult Q-Sort (CAQ: Block, 1978) contains 100 diverse personality characteristics, and each participant describes his or her own personality by placing each of the items into one of nine categories, from extremely uncharacteristic to extremely characteristic, with a fixed number of items allowed for each category. For this particular study, the CAQ was revised for cross-cultural use to make the items easier for translation by simplifying and removing English-specific jargon (Guillaume \& Funder, 2012; see Appendix A1).

\section{Procedure}

The CAQ was administered online using a custom-built website. The CAQ was translated into each country's language and then back-translated to ensure accuracy. A qual- ified bilingual individual without prior knowledge of the CAQ translated each language's version back in to English and the two were compared. Although some CAQ items could not retain their literal translations, discussions with collaborators (who are all psychologists) worked to ensure that the items retained their original meanings as closely as possible.

Next, collaborators distributed login IDs to participants within each of their countries. All participants completed the survey online, ensuring the study was implemented in the same manner across countries. Once participants logged in, they provided basic demographic information (e.g., native language, country of birth, country of childhood, mother's country of origin, father's country of origin).

Lastly, participants described their personalities using the CAQ. The 100 items were presented in random order, to avoid biases that have been found to be associated with order of presentation (Serfass \& Sherman, 2013). Once completed, participants were thanked, and the researchers' contact information was provided.

\section{RESULTS}

\section{Average CAQ mean item placements and positivity across countries}

As the first step in data analysis, we examined the overall highest and lowest mean item placements of CAQ items averaged across countries. Many analyses of personality have uncovered general tendencies toward evaluative positivity. The present data show this as well. Across all 13 countries, the most endorsed items were positive and socially desirable, and the least endorsed items were negative or undesirable in nature. For a list of items presented from most endorsed to least endorsed averaged across countries, see Appendix A2.

In terms of items placed highest across our countries, item \#56, "Responds to and appreciates humor" was among the highest rated items in 9 out of 13 countries, with means 
Table 2. Correlations of averaged CAQ profiles of women and men (combined) across 13 countries

\begin{tabular}{|c|c|c|c|c|c|c|c|c|c|c|c|c|c|}
\hline & $\mathrm{CA}$ & $\mathrm{CN}$ & $\mathrm{CZ}$ & FR & PL & RU & SG & SK & $\mathrm{ZA}$ & KR & $\mathrm{CH}$ & UK & US \\
\hline $\mathrm{CA}$ & - & & & & & & & & & & & & \\
\hline $\mathrm{CN}$ & .83 & - & & & & & & & & & & & \\
\hline $\mathrm{CZ}$ & .83 & .79 & - & & & & & & & & & & \\
\hline FR & .84 & .74 & .82 & - & & & & & & & & & \\
\hline PL & .82 & .76 & .82 & .83 & - & & & & & & & & \\
\hline RU & .84 & .69 & .77 & .81 & .79 & - & & & & & & & \\
\hline SG & .91 & .80 & .82 & .82 & .73 & .77 & - & & & & & & \\
\hline SK & .83 & .72 & .85 & .79 & .79 & .82 & .79 & - & & & & & \\
\hline $\mathrm{ZA}$ & .94 & .80 & .86 & .90 & .85 & .84 & .90 & .84 & - & & & & \\
\hline $\mathrm{KR}$ & .83 & .82 & .74 & .71 & .69 & .69 & .80 & .71 & .76 & - & & & \\
\hline $\mathrm{CH}$ & .86 & .78 & .88 & .90 & .83 & .77 & .86 & .83 & .90 & .74 & - & & \\
\hline UK & .93 & .74 & .81 & .86 & .80 & .83 & .87 & .82 & .92 & .75 & .86 & - & \\
\hline US & .98 & .83 & .84 & .85 & .83 & .83 & .92 & .83 & .94 & .81 & .87 & .91 & - \\
\hline Avg & .89 & .78 & .82 & .83 & .80 & .79 & .84 & .81 & .88 & .76 & .85 & .85 & .88 \\
\hline $95 \% \mathrm{CI}$ & $.86-.92$ & $.75-.81$ & $.80-.84$ & $.80-.86$ & $.77-.83$ & $.76-.82$ & $.81-.87$ & $.79-.83$ & $.85-.91$ & $.74-.78$ & $.83-.87$ & $.82-.88$ & $.86-.90$ \\
\hline
\end{tabular}

Note: Countries are as follows: Canada, China, Czech Republic, France, Poland, Russia, Singapore, Slovakia, South Africa, South Korea, Switzerland, UK, US. Averages computed using the $r$-to- $z$ transformation. The most similar countries (with each other and overall) are highlighted in blue; the least similar are highlighted in yellow.

ranging from 7.07 to 7.35 out of 9 . Countries where this item was rated high included Canada, the Czech Republic, France, Singapore, Slovakia, South Africa, Switzerland, the UK, and the US. The second highest-rated item, on average, appeared in 7 out of our 13 countries sampled and was item 70, "Behaves ethically; has a personal value system and is faithful to it," with mean item placements ranging from 6.6 to 7.28 .

The items rated lowest across our countries included item 36, "Tends to undermine, obstruct, or sabotage other people," which was among the top two least endorsed items in 11 out of 13 of our countries, with means ranging from 2.06 to 2.91 out of 9 . South Korea was the only country that did not have this item among the two least endorsed items. The second lowest-rated item, on average, appeared in 10 out of our 13 countries sampled and was 37, "Is guileful, deceitful, manipulative, opportunistic; takes advantage of others," with mean item placements ranging from 1.99 to 3.08. (France, Russia, and the US did not have this item among their two lowest rated).

Based on these analyses, it appears that individuals in most countries reported similar personality profiles on average, and mostly positive or socially desirable personality attributes were given the highest ratings. In order to test if items rated higher were more socially desirable, we correlated the average mean profiles of all CAQ items with an optimally adjusted character template. The template of the optimally adjusted character consisted of CAQ items that were selected by raters as the most and least ideal traits, rated on a one to nine scale. The optimally adjusted character is someone who is warm, productive, insightful, ethically consistent, perceptive, and candid. The opposite is someone who exudes hostility, anxiety, fearfulness, pervasive guilt feelings, distrust, self-pity, and the use of repressive mechanisms (Block, 1978). There was a strong positive correlation with the average CAQ profile across countries $(r=.92)$, indicating that respondents in this study report possessing more positive or desirable characteristics than negative ones, and describe themselves as generally well adjusted.

\section{Cross-cultural Similarity in CAQ profiles}

To compare the average personality profiles across countries, we separated each country's sample by gender, and then averaged all participants' Q-sorts. This yielded one average CAQ profile for each combination of gender and country, resulting in $26 \mathrm{CAQ}$ profiles. We then averaged men's and women's profiles within each country, resulting in 13 CAQ profiles, one for each country. This method allows both genders to contribute equally to the composite, instead of giving more weight to data from our larger samples of women. Although this procedure assumes that both men and women were adequately sampled in each country, certain countries had particularly low numbers of male participants in the sample (e.g., Slovakia and Poland). Thus, these results should be viewed with caution. The average Qsorts for each country were then correlated with each other. The results appear in Table 2.

In general, the averaged CAQ profiles were very similar. Across all 13 countries, the average cross-cultural correlation was $r=.82$, reflecting how the normative personality profile is similar across countries (Furr, 2008). The two countries most similar in averaged personality profiles were the United States and Canada $\left(r(98)^{\mathrm{ii}}=.98,95 \%\right.$ CI [.84, .92]). The lowest similarities were between South Korea and Poland, South Korea and Russia, and Russia and China $(r(98)=.69,[.57, .78])$.

The bottom rows of Table 2 show the average correlation of each country's mean profile with the other 12 coun-

ii Each correlation reflects the relationship between patterns of 100 average Q-sort placements in each country; hence the $d f=98$. 
Table 3. Average inter-individual CAQ correlations of women and men (combined) within and across 13 countries

\begin{tabular}{|c|c|c|c|c|c|c|c|c|c|c|c|c|c|}
\hline & $\mathrm{CA}$ & $\mathrm{CN}$ & $\mathrm{CZ}$ & FR & PL & RU & SG & SK & $\mathrm{ZA}$ & KR & $\mathrm{CH}$ & UK & US \\
\hline $\mathrm{CA}$ & .28 & & & & & & & & & & & & \\
\hline $\mathrm{CN}$ & .21 & .22 & & & & & & & & & & & \\
\hline $\mathrm{CZ}$ & .25 & .21 & .31 & & & & & & & & & & \\
\hline FR & .23 & .18 & .24 & .24 & & & & & & & & & \\
\hline PL & .25 & .20 & .26 & .24 & .28 & & & & & & & & \\
\hline RU & .23 & .17 & .22 & .22 & .23 & .25 & & & & & & & \\
\hline SG & .23 & .18 & .22 & .20 & .20 & .19 & .22 & & & & & & \\
\hline SK & .26 & .20 & .28 & .24 & .26 & .25 & .22 & .29 & & & & & \\
\hline $\mathrm{ZA}$ & .25 & .19 & .24 & .23 & .23 & .21 & .21 & .24 & .23 & & & & \\
\hline $\mathrm{KR}$ & .18 & .16 & .17 & .15 & .16 & .15 & .16 & .17 & .15 & .15 & & & \\
\hline $\mathrm{CH}$ & .25 & .21 & .27 & .25 & .26 & .22 & .22 & .27 & .24 & .17 & .28 & & \\
\hline UK & .25 & .18 & .23 & .23 & .23 & .22 & .21 & .25 & .23 & .16 & .24 & .25 & \\
\hline US & .26 & .20 & .23 & .22 & .23 & .21 & .22 & .24 & .23 & .17 & .24 & .23 & .24 \\
\hline
\end{tabular}

Note: Countries are as follows: Canada, China, the Czech Republic, Poland, Russia, Singapore, Slovakia, South Africa, South Korea, the UK, US. Diagonal figures in boldface represent within-country homogeneity; highest is highlighted in blue and lowest in yellow.

tries' mean profiles (excluding each comparison country's data from the total profiles). Confidence intervals are included around each mean correlation, and a one-way ANOVA demonstrated that the mean correlations differed significantly across countries $(F(12,143)=6.35, p<.001)$. The country most similar to all the others was Canada (average $r(12)=.89,95 \% \mathrm{CI}[.87, .91])$; the country least similar to the others was South Korea (average $r(12)=.76,[.74$, $.78])$. Overall, the finding of high similarity among average country profiles implies that the meaning of the $100 \mathrm{Q}$-sort items, relative to each other, is consistent across the 13 countries in which it was assessed.

\section{Within-country homogeneity}

The correlations described thus far are all between averages of personality profiles computed within each country. With these data, it was also possible to assess the degree of similarity of CAQ reports among individuals within each country. The correlations along the diagonal in Table 3 (highlighted in bold) can be interpreted as measures of withincountry homogeneity of personalities - the degree to which individuals tend to resemble other individuals in the same country. A higher correlation indicates greater similarity among individuals within that country. Not surprisingly, these numbers were much smaller than the correlations in Table 3, because they represent correlations among individuals rather than averaged mean profiles. By this measure, the country with the most homogeneity of personality profiles was the Czech Republic (within-country average $r(264)=.31,95 \%$ CI $[.29, .33])$, and the least homogenous was found within South Koreans (average $r(102)=.15$, [.13, .19]).

In further analyses, each participant's CAQ in each country was correlated with every other participant's (of the same gender, and then averaged men and women) in each of the other countries, and the average of these correlations is reported in the off-diagonal cells of Table 3 . The betweencountry comparisons are not dramatically smaller than the within-country comparisons. The within-country average was $r(12)=.25,95 \%$ CI $[.22, .26]$, while the across-countries average was $r(77)=.22,[.21, .23]$.

Previous research has revealed a geographic pattern in standard deviations: Asian and African countries have smaller variability in personality, and European countries have shown greater heterogeneity (McCrae, 2002). However, our data show that South Korea had the least homogeneity, and the Czech Republic showed the most. The findings in Table 3 are mixed; no clear pattern of expected differences in homogeneity between Asian, African, and European countries emerged.

\section{The CAQ Big Five dimensions}

Although the CAQ was not originally designed to measure the Big Five, in a previous study latent factors roughly analogous to the Big Five traits were identified using a varimaxrotated principal component analysis (McCrae et al., 1986).

For each individual in each country, a mean score of all the relevant CAQ Big Five items was obtained by averaging each individual's score for the given equally weighted items. Items that loaded negatively were reverse-scored. These individual scores were then averaged within country, separated by gender. The separate gender scores were then averaged within each country to create a gender balanced trait score for each country. The average trait scores and standard deviations for each country are presented in Table 4.

Agreeableness had the highest average trait score across the 13 countries $(M=5.84, S D=.67)$. Canada had the highest agreeableness score while France had the lowest score. Extraversion had the second highest average trait score (M $=5.55, \mathrm{SD}=.71)$. Poland had the highest extraversion score while Slovakia had the lowest. Neuroticism had the lowest average trait score across the 13 countries but the highest standard deviation $(\mathrm{M}=4.29, \mathrm{SD}=.81)$. South Korea had the highest neuroticism score while China had the lowest score. Openness had the lowest standard deviation $(\mathrm{M}=$ 
Table 4. CAQ Big Five scores and standard deviation by country

\begin{tabular}{lcccccccccc}
\hline Country & $\mathrm{E}$ & $(\mathrm{SD})$ & $\mathrm{N}$ & $(\mathrm{SD})$ & $\mathrm{A}$ & $(\mathrm{SD})$ & $\mathrm{C}$ & $(\mathrm{SD})$ & $\mathrm{O}$ & $(\mathrm{SD})$ \\
\hline Canada & 5.60 & .66 & 4.18 & .72 & 6.07 & .71 & 5.57 & .68 & 5.21 & .56 \\
China & 5.57 & .68 & 4.15 & .84 & 5.79 & .63 & 5.73 & .76 & 5.09 & .45 \\
Czech Republic & 5.42 & .74 & 4.22 & .81 & 5.90 & .63 & 5.82 & .71 & 5.53 & .65 \\
France & 5.49 & .81 & 4.40 & .85 & 5.63 & .69 & 5.55 & .70 & 5.63 & .59 \\
Poland & 5.69 & .73 & 4.25 & .85 & 5.75 & .63 & 5.79 & .80 & 5.42 & .59 \\
Russia & 5.50 & .67 & 4.35 & .78 & 5.72 & .67 & 5.21 & .66 & 5.52 & .62 \\
Singapore & 5.42 & .70 & 4.41 & .79 & 5.81 & .73 & 5.58 & .76 & 5.18 & .62 \\
Slovakia & 5.20 & .79 & 4.30 & .88 & 6.04 & .60 & 5.57 & .70 & 5.43 & .67 \\
South Africa & 5.43 & .70 & 4.22 & .81 & 5.72 & .66 & 5.70 & .74 & 5.52 & .65 \\
South Korea & 5.52 & .73 & 4.44 & .77 & 5.80 & .70 & 5.49 & .82 & 5.14 & .53 \\
Switzerland & 5.45 & .74 & 4.23 & .80 & 5.86 & .70 & 5.85 & .72 & 5.49 & .63 \\
United Kingdom & 5.64 & .64 & 4.38 & .86 & 5.89 & .74 & 5.37 & .65 & 5.43 & .62 \\
United States & 5.53 & .64 & 4.17 & .75 & 5.93 & .68 & 5.54 & .67 & 5.20 & .55 \\
\hline Average & 5.55 & .71 & 4.29 & .81 & 5.84 & .67 & 5.60 & .72 & 5.37 & .59 \\
\hline
\end{tabular}

Note: CAQ Big Five traits were based on principal components analyses from McCrae et al. (1986). Each country's trait score was first averaged separately for each gender to create a gender balanced mean.

$5.27, \mathrm{SD}=.59)$, and was rated highest in France and lowest in China. For conscientiousness $(\mathrm{M}=5.60, \mathrm{SD}=.72)$, Switzerland had the highest score while Russia had the lowest score. Overall, there were no clear regional patterns in trait scores, with the exception of openness that was rated lowest in all the Asian countries.

We then calculated the amount of within vs between country variability for each of the Big Five traits, using ICC(1). There was little between country variability in extraversion $(\mathrm{ICC}=.01)$, neuroticism $(\mathrm{ICC}=.01)$, and agreeableness $(\mathrm{ICC}=.04)$, and modest between country variability in conscientiousness $(\mathrm{ICC}=.04)$ and openness $(\mathrm{ICC}=.08)$. Overall, there was much greater within country variability than between country variability.

\section{Country level comparisons with previous research}

Thus far, we have explored trends in our data using the CAQ-derived Big Five, but we have not empirically com-

Table 5. Correlations between CAQ derived Big Five scores and Schmitt et. (2007) BFI country scores

\begin{tabular}{|c|c|c|c|c|c|}
\hline & $\begin{array}{l}\text { E (CAQ) } \\
{[95 \% \mathrm{CI}]}\end{array}$ & $\begin{array}{l}\text { N (CAQ) } \\
{[95 \% \mathrm{CI}]}\end{array}$ & $\begin{array}{l}\text { A (CAQ) } \\
{[95 \% \mathrm{CI}]}\end{array}$ & $\begin{array}{l}\text { C (CAQ) } \\
{[95 \% \mathrm{CI}]}\end{array}$ & $\begin{array}{l}\text { O (CAQ) } \\
{[95 \% \mathrm{CI}]}\end{array}$ \\
\hline $\begin{array}{c}\mathrm{E} \\
(\mathrm{BFI})\end{array}$ & $\begin{array}{c}-.04 \\
{[-.65, .60]}\end{array}$ & .33 & .12 & .16 & -.37 \\
\hline $\begin{array}{c}\mathrm{N} \\
(\mathrm{BFI})\end{array}$ & -.64 & $\begin{array}{c}.54 \\
{[-.14, .87]}\end{array}$ & -.45 & -.34 & -.58 \\
\hline $\begin{array}{c}\mathrm{A} \\
(\mathrm{BFI})\end{array}$ & .14 & -.09 & $\begin{array}{c}.13 \\
{[-.54, .70]}\end{array}$ & -.04 & -.09 \\
\hline $\begin{array}{c}\mathrm{C} \\
(\mathrm{BFI})\end{array}$ & .28 & -.39 & -.20 & $\begin{array}{c}-.36 \\
{[-.81, .35]}\end{array}$ & .70 \\
\hline $\begin{array}{c}\mathrm{O} \\
(\mathrm{BFI}) \\
\end{array}$ & .31 & -.27 & -.11 & .13 & $\begin{array}{c}.45 \\
{[-.25, .84]}\end{array}$ \\
\hline
\end{tabular}

pared these results with previous research. One way to examine the validity of the CAQ Big Five trait scores is to correlate the country-level means in the CAQ Big Five with country-level means in others' data. If positive relationships exist between our findings and other cross-cultural research on the Big Five, we can become more confident that the CAQ Big Five may estimate similar latent variables across countries (Campbell \& Fiske, 1959; Cronbach \& Meehl, 1955).

Our first comparison was made with data reported by Schmitt et al. (2007). Across 56 nations, Schmitt et al. (2007) provided country level $T$-scores for each of the Big Five traits assessed by the 44-item Big Five Inventory (BFI; Benet-Martínez \& John, 1998). There were 10 countries that overlapped with our CAQ data. Table 5 lists correlations between country-level average scores for each CAQ Big Five domain in our samples and the corresponding BFI domains in the Schmitt et al. (2007) samples. Neuroticism has the strongest correlation $(r=.54,95 \%$ CI [-.14, .87]), followed by openness $(r=.45,[-.25, .84])$, and agreeableness $(r=.13$ $[-.54, .70])$. Extraversion $(r=-.04[-.65, .60])$, and conscientiousness $(r=-.36[-.81, .35])$ were negatively related.

Our second comparison was with data accumulated by Allik et al. (2017) on NEO-PI-R country scores. There were 11 countries that overlapped with our CAQ data.

Table 6 lists the correlations between country-level average scores for each CAQ Big Five trait and the NEO-PI$\mathrm{R}$ traits from Allik et al., (2017). Agreeableness had the strongest correlation $(r=.44,95 \%$ CI $[-.22, .82])$, followed by extraversion $(r=.29[-.38, .76])$, and openness $(r=.25$, $[-.41, .74])$. Neuroticism $(r=-.09,[-.65, .54])$ and conscientiousness $(r=-.24,[-.73, .42])$ were negatively related.

Additionally, many of the non-corresponding traits across measures were highly correlated across countries, sometimes more so than the corresponding trait. CAQ openness was positively related to BFI conscientiousness ( $r=$ .70) while CAQ extraversion was negatively related to BFI neuroticism $(r=-.64)$. CAQ neuroticism scores were negatively related to NEO-PI-R agreeableness scores $(r=-.70)$ and positively related to NEO-PI-R openness scores $(r=$ 
Table 6. Correlations between CAQ derived Big Five scores and Allik (2017) NEO country scores

\begin{tabular}{|c|c|c|c|c|c|}
\hline & $\begin{array}{l}\text { E (CAQ) } \\
{[95 \% \mathrm{CI}]}\end{array}$ & $\begin{array}{l}\text { N (CAQ) } \\
{[95 \% \mathrm{CI}]}\end{array}$ & $\begin{array}{l}\mathrm{A}(\mathrm{CAQ}) \\
{[95 \% \mathrm{CI}]}\end{array}$ & $\begin{array}{l}\text { C (CAQ) } \\
{[95 \% \mathrm{CI}]}\end{array}$ & $\begin{array}{l}\text { O (CAQ) } \\
{[95 \% \mathrm{CI}]}\end{array}$ \\
\hline $\mathrm{E}$ & .29 & & & & \\
\hline (NEO) & {$[-.38, .76]$} & -.39 & .03 & .45 & -.38 \\
\hline $\mathrm{N}$ & & -.09 & & & \\
\hline (NEO) & -.12 & {$[-.65, .54]$} & .35 & .18 & .49 \\
\hline A & & & .4 & & \\
\hline $\begin{array}{c}\text { (NEO) } \\
\mathrm{C}\end{array}$ & .46 & -.39 & {$[-.22, .82]$} & $\begin{array}{l}.37 \\
-.24\end{array}$ & .29 \\
\hline (NEO) & -.27 & .41 & -.19 & {$[-.73, .42]$} & .09 \\
\hline $\begin{array}{c}\mathrm{O} \\
(\mathrm{NEO})\end{array}$ & .18 & .06 & -.10 & -.17 & $\begin{array}{c}.25 \\
{[-.41, .74]}\end{array}$ \\
\hline
\end{tabular}

Note: $* p<.05$ Big Five NEO scores were originally published by Allik et al. (2017). CAQ Big Five traits were based on principal components analyses from McCrae et al. (1986). There were 11 overlapping countries: Canada, China, Czech Republic, France, Poland, Russia, South Africa, South Korea, Switzerland, UK, \& US.

.55). CAQ extraversion scores were negatively correlated with NEO-PI-R neuroticism scores $(r=-.55)$ and positively correlated with NEO-PI-R agreeableness scores $(r=.50)$. Of all the Big Five traits, only neuroticism for the BFI comparisons and agreeableness for the NEO comparisons had a stronger correlation with their corresponding trait compared with their non-corresponding traits.

\section{The magnitude of gender differences in the CAQ Big Five}

Researchers have noted that gender differences in the Big Five tend to be smaller in African and Asian countries, and larger in European and North American countries. Costa et al. (2001) and Schmitt et al. (2008) both calculated the magnitude of gender differences within each of the counties in their studies. Table 7 shows the magnitude of gender differences in the CAQ derived traits with Cohen's $d$, calculated as the mean of women's z-scores subtracted from the mean of men's $z$-scores divided by their pooled standard deviation. In general, effect sizes varied greatly depending on the country and trait. Across the 13 countries, the largest differences were for CAQ agreeableness $(d=.30)$, extraversion $(d=.25)$ and neuroticism $(d=.22)$, which were on average higher in women. Only openness had a negative average $d$, indicating higher average levels in men. For agreeableness, Poland $(d=.89)$ and Switzerland $(d=.69)$ had the largest differences; China $(d=.18)$ and France $(d=.03)$ had the smallest differences. Poland $(d=-.84)$ and Switzerland $(d=$ -.41) also had the largest gender differences in openness, this time with men rating themselves higher than women. Across all five traits, Slovakia $(d=.28)$ and Poland $(d=.23)$ had the largest gender differences, while South Korea $(d=$ $.04)$ and Singapore $(d=.03)$ had the smallest gender differences.

Previous research has shown that gender differences tend to be smaller in African and Asian countries than in European counties (Costa et al., 2001; Schmitt et al., 2008). Qualitatively, the results from this sample parallel these previous findings. The Asian countries had the smallest gender differences while Western countries had the biggest differences. Schmitt et al. (2008) found these cultural differences in gender differences could be attributed to the country's economic and social development. To test this with our data, we used Multilevel Modeling (MLM) to determine if a country's Human Development Index (HDI) rating affected the degree to which gender predicted each personality trait. There was a significant interaction between HDI and gender predicting neuroticism $(\beta=-1.07, t=-2.45, p=.01)$ and conscientiousness $(\beta=1.39, t=3.47, p<.001)$, but not for extraversion $(\beta=-.56, t=-1.45, p=.15)$, agreeableness $(\beta$ $=-.29, t=-.81, p=.42)$, or openness $(\beta=.60, t=1.90, p=$ $.06)$. Thus, for countries with higher levels of development, there were less gender differences in neuroticism but greater gender differences in conscientiousness.

\section{DISCUSSION}

The purpose of this study was to compare personality measured by the California Adult Q-set (CAQ) and the Big Five factors derived from the CAQ across multiple countries. Cross-cultural research on personality has primarily utilized Likert scales which have been dogged by issues related to response biases, social desirability, and reference group effects (Chen, 2008; Cheung \& Rensvold, 2000; Heine et al., 2002; Ross \& Mirowsky, 1984; van de Vijver, \& Leung, 2011). Researchers have suggested that forced choice measures such as the CAQ may help to circumvent issues with nomothetic Likert scales, and serve as an alternative method for examining personality across countries (Heine et al., 2002; Marsella, Dubanoski, Hamada, \& Morse, 2000). The 100-item California Adult Q-set (CAQ) provided a number of ways to examine personality around the world and some convergences as well as discrepancies with previous findings. Overall, results from this study indicate forced-choice assessments of personality traits produce moderately similar results when compared with traditional Likert-type questionnaires.

\section{Averaged CAQ profiles and inter-individual profiles}

The overall self-reported personality profiles of individuals around the world were, on average, highly similar and largely positive. The homogeneity of personalities was nearly as pervasive between countries as within. While this amount of similarity may or may not be surprising, it is a further encouraging indication, along with the correlations among the inter-item matrices, that the content of the CAQ items were indeed understood similarly across several different countries and languages.

The degree of similarity of CAQ reports among individuals within each country revealed that South Korea was the least homogenous country, and the Czech Republic the most. The homogeneity of personality profiles was nearly as great between countries as within. Although smaller than the within-country comparisons, the between-country comparisons were not dramatically smaller. This finding suggests a greater degree of similarity in personality traits around the world than perhaps would have been expected before cross- 
Table 7. Mean z score differences (d) between women and men in 13 countries on CAQ Big Five factors

\begin{tabular}{|c|c|c|c|c|c|c|}
\hline & $\mathrm{E}$ & $\mathrm{N}$ & $\mathrm{A}$ & $\mathrm{C}$ & $\mathrm{O}$ & Average \\
\hline Canada & -.01 & .44 & .33 & -.05 & -.14 & .11 \\
\hline China & .07 & -.26 & .18 & .31 & .07 & .07 \\
\hline Czech Republic & .32 & .27 & .28 & .09 & -.28 & .14 \\
\hline France & .16 & .36 & .03 & -.18 & .13 & .10 \\
\hline Poland & .27 & .40 & .89 & .42 & -.84 & .23 \\
\hline Russia & .35 & .48 & .35 & -.24 & .09 & .21 \\
\hline Singapore & .08 & .13 & .23 & -.38 & .11 & .03 \\
\hline Slovakia & .84 & .14 & .25 & .50 & -.31 & .28 \\
\hline South Africa & .01 & .19 & .27 & .12 & .14 & .15 \\
\hline South Korea & .18 & .40 & -.26 & -.85 & .34 & -.04 \\
\hline Switzerland & .71 & -.14 & .69 & -.04 & -.41 & .16 \\
\hline United Kingdom & .09 & .22 & .21 & .03 & -.34 & .04 \\
\hline United States & .19 & .22 & .43 & -.04 & -.08 & .14 \\
\hline Average & .25 & .22 & .30 & .02 & -.12 & \\
\hline
\end{tabular}

Note: For each country, women's means were subtracted from men's; negative $d$ 's indicate that the men's mean was larger than the women's mean.

cultural research began, but is consistent with results reported by other investigators (McCrae, 2002; Allik et al., 2017).

\section{Highest and lowest CAQ mean item placements across 13 countries}

The similarities of CAQ profiles across countries showed that the most highly rated items were similarly positive across countries, and the lowest rated items were negative. High average ratings of items such as "responds to and appreciates humor," and "behaves ethically; has a personal value system and is faithful to it," indicate that people describe themselves primarily in positive terms. Likewise, the lowest-rated items of "tends to undermine, obstruct, or sabotage other people," and "is guileful, deceitful, manipulative, opportunistic; takes advantage of others," indicate that people generally do not consider themselves to possess primarily undesirable traits. This trend was noted across all 13 countries sampled in this study.

\section{Country differences in the CAQ Big Five}

North American and European countries tended to have the highest extraversion scores, and South Africa and Asian countries had the lowest extraversion scores. For CAQ neuroticism, North American countries tended to have the lowest neuroticism scores, and East Asian countries the highest. For CAQ agreeableness, Canada had the highest scores on average, and France the lowest. The East Asian countries in our sample (Singapore, South Korea, and China) were neither high nor low in agreeableness. These findings are surprising, because one might predict that countries that value harmony and in-group norms would score high in agreeableness, whereas those less concerned with getting along and perhaps more concerned with getting ahead would score low in agreeableness (Triandis, 1997).

For CAQ conscientiousness, Switzerland had the highest scores on average, and Russia the lowest. Although widely- held stereotypes maintain that Asians are prototypically industrious and detail-oriented (Mõttus et al., 2012) the present study shows that China and Singapore are just about average in conscientiousness among our 13 countries, and South Korea had the third lowest CAQ conscientiousness scores. Recent studies have also found that Asian countries score at or below average (McCrae et al., 2005, Schmitt et al., 2007). This distinction could reflect more of a cultural difference in self-enhancement than actual trail-level variation, as Westerns have been found to have a higher self-serving bias than East Asians (Heine \& Hamamura, 2001).

For CAQ openness, European countries tended to have the highest scores, followed by Canada and the US, and then East Asian countries. We found that France was the highest in openness, and China the least. Allik and McCrae (2004) also found that Europeans and Americans are more open to new experiences, whereas East Asian and African countries are more traditional.

\section{Gender differences in the CAQ Big Five}

Previous researchers have found that gender differences in the Big Five personality traits or facets have a geographically ordered pattern, with the largest gender differences found in Europe and the smallest differences in East Asian and African countries (Costa et al., 2001; Schmitt et al., 2008). In general, the results from this study trended similarly with previous findings: South African and East Asian samples had smaller gender differences than our European and North American samples across the CAQ Big Five. Additionally, the results of this study confirmed previous findings on individual trait-level differences. On average, women scored higher on neuroticism, extraversion, and agreeableness.

Previous researchers have speculated these gender differences may be due to social desirable responding or the reference group effect. However, the CAQ instrument helps decrease the influence of these effects, indicating that our results may reflect some underlying difference. Schmitt et al. (2008) speculated that the bigger gender differences in 
Western countries may be a reflection of greater freedom for individual expression. In countries with more equality of resources and opportunities, individual differences might have a greater impact on behavior. Gender differences in personality could be suppressed in less developed nations with less equality of resources. However, the results of the MLM analyses were inconsistent. Countries with higher levels of development had smaller gender differences in neuroticism but greater differences in conscientiousness. Results for the other 3 traits were not significant. One potential explanation is the limited variability in HDI scores for this sample. The non-Western sample included countries such as South Korea and Singapore, which are very wealthy compared to many other Asian and African countries included in previous cross-cultural research on gender differences in personality traits. Thus, while all the East Asian countries included in this sample exhibited some of the smallest gender differences in personality traits, these differences, or lack thereof, could not necessarily be attributed to their economic development.

\section{Country-level correlations}

At the country level, mean differences CAQ-derived Big Five scores were compared to those reported from earlier studies using the NEO-PI-R (Allik et al., 2017) and BFI traits (Schmitt et al., 2008), finding moderate agreement across samples and measures. For the NEO-PI-R scores, the highest correlation with a CAQ trait was neuroticism, followed by openness and agreeableness. Extraversion scores were unrelated to each other and conscientiousness scores had a negative correlation across the two studies. Similar to the results with the NEO-PI-R, CAQ derived neuroticism scores and BFI neuroticism country scores were also strongly related. Country-level openness scores also produced similar agreement across measures. Extraversion, conscientiousness and agreeableness scores were unrelated between the CAQ derived country scores and the BFI country scores.

Additionally, many of the correlations between non-corresponding traits were high, some even higher than the corresponding trait. For example, CAQ derived neuroticism had a strong negative correlation with NEO-PI-R agreeableness scores that was higher than the correlation between CAQ derived agreeableness scores and NEO-PI-R agreeableness scores. And while CAQ derived extraversion scores were unrelated to NEO-PI-R extraversion scores, they were positively correlated with NEO-PI-R agreeableness scores and negatively correlated with NEO-PI-R neuroticism scores. However, these unexpected correlations between non-corresponding personality traits are consistent with a recent review by Allik and Realo (2017) that found higher correlations between some non-corresponding traits between BFI country scores (Schmit et al., 2007) and NEOPI-R country scores (Allik \& McCrae, 2004) than corresponding traits.

Overall, despite differences in measures, methods, time of collection, researchers, and sampling strategies, the country level means for the CAQ neuroticism and openness factors show similarities to the findings of Allik et al. (2017) and of Schmitt et al. (2007), just as other researchers found strong correlations between different instruments that measure the Big Five (McCrae, 2002). However, the remaining country-level traits of conscientiousness, agreeableness, and extraversion were not related across measures, indicating only moderate stability of country-level trait scores overall.

\section{Limitations}

The number of countries sampled in this study is relatively small, compared with other cross-cultural assessments of personality, and certain samples within countries were also limited. Only university students with internet access were included, therefore we cannot claim they were representative of any country's overall population. Additionally, the students sampled in this study described their personalities in mostly similar, positive terms, which suggests that global university cultures may share certain commonalties that affect aspects of personality or vice versa (Flere \& Lavrič, 2008). Therefore, this survey would need to be administered to members of the larger community before the findings may be generalized to the greater populations of each country. Lastly, the CAQ may be considered an imposed etic, as its 100 items were developed in the US and did not originally include culturally specific personality characteristics. While the CAQ items - like the Big Five traits - could be considered comprehensive for a Western sample, the same may not hold true for non-WEIRD populations.

\section{Conclusion}

This was an exploratory study that utilized the California Adult Q-set (CAQ) to examine the similarities in personality profiles across cultures, and to compare the CAQ Big Five with previous cross-cultural findings in personality by a) recruiting similar samples, using similar methods across countries (avoiding sample bias), b) using a personality measure that examines single-item personality characteristics across countries without incorporating complex constructs (avoiding problems of replication and construct bias), c) administering a forced-choice instrument instead of a Likert scale (avoiding instrument biases and reference group effects), and d) correlating the CAQ Big Five results with other results found in the literature on the Big Five as measured across cultures (testing for cross-instrument validity).

Before the development of our website, it was extremely difficult to run online cross-cultural studies using a forcedchoice instrument, yet researchers have speculated about the potential usefulness of forced-choice measures in cross-cultural research for years (Heine et al., 2002). Do Q-sorts yield dramatically different results from the Likert scales that have traditionally been used to measure personality in crosscultural research? Overall, the present findings suggest the answer is no. Findings from this study show convergent validity with previous research on the Big Five across countries (McCrae et al., 2005; Schmitt et al., 2007), and several trends within the CAQ data converged with cross-cultural research on gender and country differences (Allik \& McCrae, 2002; Allik \& McCrae, 2004; Costa et al., 2001; McCrae, 2004; McCrae et al., 2005). Simply put, if Q-sorts provided drastically different results from Likert scales, the CAQ Big Five would not have aligned with other measures 
of the Big Five. As always, this study and others like it will need to be replicated. However, the present results suggest that on average, people - our personalities - are more similar around the world than we may have expected more than 50 years ago when the study of personality had just begun.

\section{NOTES AND ACKNOWLEDGMENT}

This study is based, in part, on the Ph.D. dissertation by Esther Guillaume at the University of California, Riverside.

The research was supported, in part, by the National Science Foundation under Grant No. BCS-1528131. Any opinions, findings, and conclusions or recommendations expressed in this material are those of the individual researchers and do not necessarily reflect the views of the National Science Foundation.

\section{REFERENCES}

Allik, J., Church, A.T.. Ortiz, F.A., Rossier, J., Hřebíčková, de Fruyt, F., Realo, A., \& McCrae, R.R. (2017). Mean profiles of the NEO Personality Inventory. Journal of Cross Cultural Psychology, 48, 402-420.

Allik, J., \& McCrae, R. R. (2002). A five-factor theory perspective. In Allik, J., \& McCrae, R. R. (Eds.), The five-factor model of personality across cultures (303-320). New York, NY: Springer. Allik, J., \& McCrae, R. R. (2004). Toward a geography of personality traits: Patterns of profiles across 36 cultures. Journal of Cross Cultural Psychology, 35, 13-28.

Allik, J., \& Realo, A. (2017). How valid are culture-level mean personality scores? In T.A. Church (Ed.), The Praeger handbook of personality across cultures, Vol. 1: Trait psychology across cultures (pp. 193-224). Santa Barabara, CA: Praeger.

Bem, D.J., \& Funder, D.C. (1978). Predicting more of the people more of the time: Assessing the personality of situations. Psychological Review, 85, 485-501.

Benet-Martínez, V., \& John, O. P. (1998). Los Cinco Grandes across cultures and ethnic groups: Multitrait-multimethod analysis of the Big Five in Spanish and English. Journal of Personality and Social Psychology, 75, 729-750.

Block, J. (1978). The Q-sort method in personality assessment and psychiatric research. Palo Alto, CA: Consulting Psychologists Press (Originally published 1961).

Campbell, D. T., \& Fiske, D. W. (1959). Convergent and discriminant validation by the multitrait-multimethod matrix. Psychological Bulletin, 56, 81-105.

Cheung, G.W., \& Rensvold, R.B (2000). Assessing extreme and acquiesce response sets in cross-cultural research using structural equations modeling. Journal of Cross-Cultural Psychology, $31,187-212$.

Chen, F. (2008). What happens if we compare chopsticks with forks? The impact of making inappropriate comparisons in crosscultural research. Journal of Personality and Social Psychology, 95, 1005-1018.

Church, A. T., Alvarez, J. M., Mai, N. T. Q., French, B. F., Katigbak, M. S., \& Ortiz, F. A. (2011). Are cross-cultural comparisons of personality profiles meaningful? Differential item and facet functioning in the revised NEO personality inventory. Journal of Personality and Social Psychology, 101, 1068-1089.

Costa, P. T., Jr., \& McCrae, R. R. (1992). Revised NEO Personality Inventory (NEO-PI-R) and NEO Five-Factor Inventory (NEO-FFI) professional manual. Odessa, FL: Psychological Assessment Resources.
Costa, P.T., Terracciano, A., \& McCrae, R. R. (2001). Gender differences in personality traits across cultures: Robust and surprising findings. Journal of Personality and Social Psychology, 81, 322-331.

Cronbach, L. J., \& Meehl, P.E. (1955). Construct validity in psychological tests. Psychological Bulletin, 52, 281-302.

Digman, J. M. (1990). Personality structure: Emergence of the five-factor model. Annual Review of Psychology, 41, 417-440.

Flere, S., \& Lavrič, M. (2008). On the validity of cross-cultural social studies using student samples. Field Methods, 20, 399412.

Furr, R. M. (2008). A framework for profile similarity: Integrating similarity, normativeness, and distinctiveness. Journal of Personality, 76, 1267-1316.

Goldberg, L. R. (1992). The development of markers for the BigFive factor structure. Psychological Assessment, 4, 26-42.

Guillaume E., \& Funder, D. (2012) CAQ Form III-R (Riverside Revised). Retrieved January 17, 2019 from https://www.situationslab.com/s/CAQ-Revised.pdf.

Heine, S. J., Lehman, D. R., Peng, K., \& Greenholtz, J. (2002). What's wrong with cross-cultural comparisons of subjective Likert scales? The reference-group effect. Journal of Personality and Social Psychology, 82, 903-918.

Heine, S. J.. Hamamura, T. (2001). Self as cultural product: An examination of East Asian and North American selves. Journal of Personality, 69, 881-905.

Marsella, A. J., Dubanoski, J., Hamada, W. C., \& Morse, H. (2000). The measurement of personality across cultures: Historical, conceptual, and methodological issues and considerations. American Behavioral Scientist, 44, 41-6

McCrae, R. R. (2001). Trait psychology and culture: Exploring intercultural comparisons. Journal of Personality, 69, 819-846.

McCrae, R. R. (2002). NEO-PI-R data from 36 cultures: Further intercultural comparisons. In R. R.McCrae \& J. Allik (Eds.), The five-factor model of personality across cultures (pp. 105-125). New York: Kluwer Academic/Plenum.

McCrae, R. R., Costa, P. T., \& Busch, C. M. (1986). Evaluating comprehensiveness in personality systems: The California Q-Set and the five-factor model. Journal of Personality, 54, 430-446.

McCrae, R. R., \& Costa, P. T. (1997). Personality trait structure as a human universal. American Psychologist, 52, 509-516.

McCrae, R. R., Terracciano, A., \& 79 Members of the Personality Profiles of Cultures Project (2005). Personality profiles of cultures: Aggregate personality traits. Journal of Personality and Social Psychology, 89, 407-425.

Mõttus, R., Allik, J., Realo, A., Pullmann, H., Rossier, J., Zecca, G., ... Tseung, C. N. (2012). Comparability of self-reported conscientiousness across 21 countries, European Journal of Personality, 317, 303-317.

Ross, C. E., \& Mirowsky, J. (1984). Socially-desirable response and acquiescence in a cross-cultural survey of mental health. Journal of Health and Social Behavior, 25, 189-197.

Schmitt, D. P., Allik, J., McCrae, R. R., Benet-Martinez, V., et al. (2007). The geographic distribution of Big Five personality traits: Patterns and profiles of human self-description across 56 nations. Journal of Cross- Cultural Psychology, 38, 173-212.

Schmitt, D. P., Realo, A., Voracek, M., \& Allik, J. (2008). Why can't a man be more like a woman? Sex differences in Big Five personality traits across 55 cultures. Journal of Personality and Social Psychology, 94, 168-182.

Serfass, D. G., \& Sherman, R. A. (2013). A methodological note on ordered Q-Sort ratings. Journal of Research in Personality, $47,853-858$.

Smith, P. B. (2004). Acquiescent response bias as an aspect of cultural communication style. Journal of Cross-Cultural Psychology, 35, 50-61.

Triandis, H.C. (1997). Cross-cultural perspectives on personality. In R. Hogan, J. A. Johnson, Briggs, \& S. R. (Eds). Handbook of personality psychology. San Diego, CA: Academic Press. 
van de Vijver, F. J. R., \& Leung, K. (1997). Methods and data analysis for cross-cultural research. Thousand Oaks, CA: Sage Publications.

van de Vijver, F. J. R., \& Leung, K. (2001). Personality in cultural context: Methodological issues. Journal of Personality, 69, 1007-1031.

van de Vijver, F. J. R., \& Leung, K. (2011). Equivalence and bias: A review of concepts, models, and data-analytic procedures. In F.J.R. Van de Vijver, \& D. Matsumoto (Eds.), Cross-cultural research methods in psychology. New York, NY: Cambridge University Press.

Received October 3, 2018

Accepted February 2, 2019 


\section{APPENDIX}

Table A1. California Adult Q-Sort (revised for cross-cultural use) Full Item Content

1. Is critical, skeptical, not easily impressed.

2. Is dependable and responsible (low placement implies undependable and irresponsible).

3. Has a wide range of interests (regardless of how deep or superficial the interests are).

4. Is a talkative person.

5. Is giving, generous toward others (regardless of the motivation).

6. Is fastidious, meticulous, careful and precise.

7. Favors conservative values in a variety of areas; emphasizes traditional values and beliefs (low placement implies rejection of traditional values).

8. Appears to have a high degree of intellectual capacity (whether or not this capacity translates into actual accomplishments).

9. Is uncomfortable with uncertainty and complexity.

10. Develops physical symptoms in reaction to stress and anxiety (e.g., sweating, racing heart, headaches, stomach aches, rashes, asthma, etc.).

11. Is protective of those close to him/her (high placement implies overprotective; medium placement implies appropriate caring; low placement implies lack of concern)

12. Tends to be self-defensive; unable to acknowledge personal shortcomings or failures; quick to defend self from criticism

13. Takes offense easily; is sensitive to anything that can be construed as a criticism or insult.

14. Genuinely submissive; accepts domination comfortably; gives in easily.

15. Is skilled in social techniques of imaginative play, pretending and humor.

16. Is introspective; thinks about self; examines own thoughts and feelings (does not necessarily imply that the person understands himself/herself well).

17. Behaves in a sympathetic and considerate manner (low placement implies unsympathetic and inconsiderate behavior).

18. Initiates humor; makes spontaneous funny remarks.

19. Seeks reassurance from others (high placement implies lack of self-confidence).

20. Behaves and acts quickly.

21. Arouses nurturant feelings in others; behaves in ways that lead others to feel caring and protective toward him/her.

22. Feels a lack of meaning in life.

23. Tends to blame others for own mistakes, failures, and shortcomings.

24. Prides self on being rational, logical and objective (high placement implies a person who is more comfortable with intellectual concepts than with feelings; low placement implies a person who is irrational and overly emotional).

25. Has excessive self-control; postpones pleasures unnecessarily.

26. Is productive; gets things done.

27. Is condescending toward others; acts superior to others.

28. Tends to arouse liking and acceptance in people (low placement implies a tendency to arouse dislike and rejection).

29. Is turned to or sought out for advice and reassurance.

30. Gives up and withdraws when possible in the face of frustration and adversity (high placement implies person gives up easily; low placement implies person does not know when, realistically, it is time to give up).

31. Regards self as physically attractive (this item refers to how person sees himself/herself, whether accurate or not).

32. Seems to be aware of the impression he/she makes on others (low placement implies person is unaware of the impression he/she makes).

33. Is calm, relaxed in manner.

34. Is irritable; overreacts to minor frustrations.

35. Has warmth; has the capacity for close relationships; compassionate.

36. Tends to undermine, obstruct, or sabotage other people.

37. Is guileful, deceitful, manipulative, opportunistic; takes advantage of others.

38. Has hostility toward others (whether or not the hostile feelings are actually expressed).

39. Thinks about ideas in unusual ways; has unconventional thought processes.

40. Is generally fearful; is vulnerable to real or imagined threat.

41. Makes moral judgments; judges self and others in terms of right and wrong (regardless of the nature of the moral code, whether traditional or liberal; high placement implies being moralistic and self-righteous; low placement implies an unwillingness to make value judgments).

42. Reluctant to commit self to any definite course of action; tends to delay or avoid making decisions or taking action.

43. Has large or vivid facial expressions or gestures.

44. Evaluates the motives of others; tries to figure out the intentions underlying people's actions (accuracy is not assumed).

45. Is psychologically frail, vulnerable; has poor ability to cope with stress.

46. Tends to fantasize and daydream.

47. Has a readiness to feel guilty (high placement implies a tendency to feel guilt even when he/she is not at fault).

48. Keeps people at a distance; avoids close relationships.

49. Is basically distrustful of people in general; questions their motivations.

50. Is unpredictable and changeable in attitudes and behavior.

51. Places high value on intellectual and cognitive matters (does not necessarily imply high intellectual achievement or intellectual ability).

52. Behaves in an assertive fashion; not afraid to express opinions; speaks up to get what he/she wants.

53. Is impulsive; has little self-control; unable to postpone pleasure. 
Table A1 continued

54. Is sociable, gregarious; emphasizes being with others.

55. Is self-defeating; acts in ways that frustrate, hurt, or undermine own chances to get what he/she wants.

56. Responds to and appreciates humor.

57. Is an interesting, colorful person.

58. Appears to enjoy sensuous experiences (e.g., touch, taste, smell, bodily contact).

59. Is concerned about own body, its health and adequacy of functioning (high placement implies excessive concern or hypochondriasis).

60. Has insight into and understands own needs, motives and behavior; knows self well (low placement implies little insight into own motives and behavior).

61. Likes others to be dependent on him/her; likes to be thought needed by others (low placement implies encouraging others to be independent of him/her).

62. Tends to be rebellious and nonconforming.

63. Is influenced by social pressures (e.g., "popularity," conventional social norms).

64. Is socially perceptive; is alert to cues from other people that reveal what they are thinking and feeling.

65. Resists limits and rules; sees what he/she can get away with.

66. Enjoys aesthetic impressions; is aesthetically sensitive (appreciates art, music, drama, etc.).

67. Is self-indulgent; tends to pamper himself or herself.

68. Is basically anxious.

69. Is sensitive to anything that can be construed as a demand or request for favors; is quick to feel imposed on.

70. Behaves ethically; has a personal value system and is faithful to it.

71. Is ambitious; sets high personal goals.

72. Has doubts about own adequacy as a person; appears to have feelings of inadequacy.

73. Tends to see sexual overtones in many situations (high placement implies reading sexual meanings into situations in which none exist; low placement implies inability to recognize sexual signals).

74. Feels satisfied with self; is unaware of self-concern

75. Is easy to understand and describe (low placement implies someone who is difficult to understand and describe).

76. Imagines that the needs, wishes and feelings of others are the same as his/her own; tends to project own feelings and motivations onto others.

77. Appears straightforward, candid, frank in dealing with others.

78. Feels cheated and victimized by life; self-pitying; feels sorry for self.

79. Tends to ruminate and have persistent, preoccupying thoughts.

80. Is sexually interested in others (whether of the opposite sex or same sex; low placement implies an absence of sexual interest).

81. Is physically attractive; is good looking (as defined by the relevant culture).

82. Has fluctuating moods; moods go up and down.

83. Able to see to the heart of important problems; does not get caught up or sidetracked by irrelevant details.

84. Is cheerful, happy (low placement implies depression).

85. Tends to communicate through actions, deeds, and non-verbal behavior, rather than through words.

86. Denies the presence of anxiety and conflicts; tends to convince himself/herself that unpleasant thoughts and feelings do not exist; deceives self into thinking everything is fine, when everything is not fine.

87. Tends to interpret clear-cut, simple situations in complicated ways.

88. Is personally charming.

89. Compares self with others; is alert to real or imagined differences between self and others in status, appearance, achievement, abilities, and so forth.

90. Is concerned with philosophical problems, for example, religions, values, free will, the meaning of life, and so forth.

91. Values power in self and others.

92. Has social poise and presence; appears socially at ease.

(a) Behaves in a masculine style or manner

(b) Behaves in a feminine style or manner

93. (If person is male, rate 93a; if person is female, rate 93b. The cultural definition of masculinity and femininity are intended here.)

94. Expresses hostility and angry feelings directly (low placement implies someone who is unable to express hostility, who holds angry feelings in).

95. Gives advice; concerns self with the business of others.

96. Values own independence and autonomy; emphasizes his/her freedom to think and act without interference or help from others.

97. Is an unemotional person; tends not to experience strong emotions (low placement implies a highly emotional person).

98. Is verbally fluent; can express ideas well in words.

99. Is self-dramatizing; theatrical; prone to exaggerate feelings; seeks attention.

100. Relates to everyone in the same way (low placement implies a person who acts differently with different people). 
Table A2. California Adult Q-sort (CAQ) highest to lowest mean item placements across 13 countries

\begin{tabular}{|c|c|c|}
\hline CAQ \# & CAQ item & Overall Mean \\
\hline 56 & Responds to and appreciates humor. & 6.98 \\
\hline 70 & Behaves ethically; has a personal value system and is faithful to it. & 6.88 \\
\hline 96 & $\begin{array}{l}\text { Values own independence and autonomy; emphasizes his/her freedom to think and act without inter- } \\
\text { ference or help from others. }\end{array}$ & 6.77 \\
\hline 51 & $\begin{array}{l}\text { Places high value on intellectual and cognitive matters (does not necessarily imply high intellectual } \\
\text { achievement or intellectual ability). }\end{array}$ & 6.56 \\
\hline 66 & Enjoys aesthetic impressions; is aesthetically sensitive (appreciates art, music, drama, etc.). & 6.44 \\
\hline 64 & Is socially perceptive; is alert to cues from other people that reveal what they are thinking and feeling. & 6.35 \\
\hline 95 & Gives advice; concerns self with the business of others. & 6.30 \\
\hline 84 & Is cheerful, happy (low placement implies depression). & 6.23 \\
\hline 71 & Is ambitious; sets high personal goals. & 6.21 \\
\hline 58 & Appears to enjoy sensuous experiences (e.g., touch, taste, smell, bodily contact). & 6.16 \\
\hline 35 & Has warmth; has the capacity for close relationships; compassionate. & 6.15 \\
\hline 60 & $\begin{array}{l}\text { Has insight into and understands own needs, motives and behavior; knows self well (low placement } \\
\text { implies little insight into own motives and behavior). }\end{array}$ & 6.11 \\
\hline 80 & $\begin{array}{l}\text { Is sexually interested in others (whether of the opposite sex or same sex; low placement implies an } \\
\text { absence of sexual interest). }\end{array}$ & 6.10 \\
\hline 77 & Appears straightforward, candid, frank in dealing with others. & 6.08 \\
\hline 16 & $\begin{array}{l}\text { Is introspective; thinks about self; examines own thoughts and feelings (does not necessarily imply } \\
\text { that the person understands himself/herself well). }\end{array}$ & 6.03 \\
\hline 17 & $\begin{array}{l}\text { Behaves in a sympathetic and considerate manner (low placement implies unsympathetic and incon- } \\
\text { siderate behavior). }\end{array}$ & 5.94 \\
\hline 98 & Is verbally fluent; can express ideas well in words. & 5.91 \\
\hline 2 & Is dependable and responsible (low placement implies undependable and irresponsible). & 5.90 \\
\hline 89 & $\begin{array}{l}\text { Compares self with others; is alert to real or imagined differences between self and others in status, } \\
\text { appearance, achievement, abilities, and so forth. }\end{array}$ & 5.89 \\
\hline 44 & $\begin{array}{l}\text { Evaluates the motives of others; tries to figure out the intentions underlying people's actions (accu- } \\
\text { racy is not assumed). }\end{array}$ & 5.85 \\
\hline 57 & Is an interesting, colorful person. & 5.84 \\
\hline 8 & $\begin{array}{l}\text { Appears to have a high degree of intellectual capacity (whether or not this capacity translates into } \\
\text { actual accomplishments). }\end{array}$ & 5.83 \\
\hline 79 & Tends to ruminate and have persistent, preoccupying thoughts. & 5.83 \\
\hline 46 & Tends to fantasize and daydream. & 5.83 \\
\hline 83 & $\begin{array}{l}\text { Able to see to the heart of important problems; does not get caught up or sidetracked by irrelevant } \\
\text { details. }\end{array}$ & 5.78 \\
\hline 88 & Is personally charming. & 5.74 \\
\hline 18 & Initiates humor; makes spontaneous funny remarks. & 5.73 \\
\hline 90 & $\begin{array}{l}\text { Is concerned with philosophical problems, for example, religions, values, free will, the meaning of } \\
\text { life, and so forth. }\end{array}$ & 5.70 \\
\hline 3 & Has a wide range of interests (regardless of how deep or superficial the interests are). & 5.67 \\
\hline 11 & $\begin{array}{l}\text { Is protective of those close to him/her (high placement implies overprotective; medium placement } \\
\text { implies appropriate caring; low placement implies lack of concern) }\end{array}$ & 5.66 \\
\hline 59 & $\begin{array}{l}\text { Is concerned about own body, its health and adequacy of functioning (high placement implies exces- } \\
\text { sive concern or hypochondriasis). }\end{array}$ & 5.66 \\
\hline 26 & Is productive; gets things done. & 5.63 \\
\hline 92 & Has social poise and presence; appears socially at ease. & 5.63 \\
\hline 54 & Is sociable, gregarious; emphasizes being with others. & 5.61 \\
\hline 28 & $\begin{array}{l}\text { Tends to arouse liking and acceptance in people (low placement implies a tendency to arouse dislike } \\
\text { and rejection). }\end{array}$ & 5.60 \\
\hline 33 & Is calm, relaxed in manner. & 5.59 \\
\hline 24 & $\begin{array}{l}\text { Prides self on being rational, logical and objective (high placement implies a person who is more } \\
\text { comfortable with intellectual concepts than with feelings; low placement implies a person who is } \\
\text { irrational and overly emotional). }\end{array}$ & 5.53 \\
\hline 29 & Is turned to or sought out for advice and reassurance. & 5.49 \\
\hline 5 & Is giving, generous toward others (regardless of the motivation). & 5.48 \\
\hline 81 & Is physically attractive; is good looking (as defined by the relevant culture). & 5.47 \\
\hline 93 & Behaves in a masculine/feminine style or manner & 5.47 \\
\hline 32 & $\begin{array}{l}\text { Seems to be aware of the impression he/she makes on others (low placement implies person is una- } \\
\text { ware of the impression he/she makes). }\end{array}$ & 5.39 \\
\hline 52 & Behaves in an assertive fashion; not afraid to express opinions; speaks up to get what he/she wants. & 5.21 \\
\hline 39 & Thinks about ideas in unusual ways; has unconventional thought processes. & 5.21 \\
\hline 41 & $\begin{array}{l}\text { Makes moral judgments; judges self and others in terms of right and wrong (regardless of the nature } \\
\text { of the moral code, whether traditional or liberal; high placement implies being moralistic and self- } \\
\text { righteous; low placement implies an unwillingness to make value judgments). }\end{array}$ & 5.18 \\
\hline
\end{tabular}


Table A2 continued

6 Is fastidious, meticulous, careful and precise.

15 Is skilled in social techniques of imaginative play, pretending and humor.

Tends to communicate through actions, deeds, and non-verbal behavior, rather than through words.

Values power in self and others.

Seeks reassurance from others (high placement implies lack of self-confidence).

Regards self as physically attractive (this item refers to how person sees himself/herself, whether accurate or not).

$4 \quad$ Is a talkative person.

20 Behaves and acts quickly.

82 Has fluctuating moods; moods go up and down.

74 Feels satisfied with self; is unaware of self-concern.

61 Likes others to be dependent on him/her; likes to be thought needed by others (low placement implies encouraging others to be independent of him/her).

47 Has a readiness to feel guilty (high placement implies a tendency to feel guilt even when he/she is not at fault).

43 Has large or vivid facial expressions or gestures.

75 Is easy to understand and describe (low placement implies someone who is difficult to understand and describe).

Has doubts about own adequacy as a person; appears to have feelings of inadequacy.

Is uncomfortable with uncertainty and complexity.

10 Develops physical symptoms in reaction to stress and anxiety (e.g., sweating, racing heart, headaches, stomach aches, rashes, asthma, etc.)

1 Is critical, skeptical, not easily impressed.

87 Tends to interpret clear-cut, simple situations in complicated ways.

42 Reluctant to commit self to any definite course of action; tends to delay or avoid making decisions or taking action.

73 Tends to see sexual overtones in many situations (high placement implies reading sexual meanings into situations in which none exist; low placement implies inability to recognize sexual signals).

69 Is sensitive to anything that can be construed as a demand or request for favors; is quick to feel imposed on.

67 Is self-indulgent; tends to pamper himself or herself.

21 Arouses nurturant feelings in others; behaves in ways that lead others to feel caring and protective toward him/her.

$7 \quad$ Favors conservative values in a variety of areas; emphasizes traditional values and beliefs (low placement implies rejection of traditional values).

63 Is influenced by social pressures (e.g., "popularity," conventional social norms).

65 Resists limits and rules; sees what he/she can get away with.

13 Takes offense easily; is sensitive to anything that can be construed as a criticism or insult

100 Relates to everyone in the same way (low placement implies a person who acts differently with different people).

76 Imagines that the needs, wishes and feelings of others are the same as his/her own; tends to project own feelings and motivations onto others.

49 Is basically distrustful of people in general; questions their motivations.

12 Tends to be self-defensive; unable to acknowledge personal shortcomings or failures; quick to defend self from criticism

68 Is basically anxious.

34 Is irritable; overreacts to minor frustrations.

25 Has excessive self-control; postpones pleasures unnecessarily.

30 Gives up and withdraws when possible in the face of frustration and adversity (high placement implies person gives up easily; low placement implies person does not know when, realistically, it is time to give up).

$48 \quad$ Keeps people at a distance; avoids close relationships.

5.16

5.09

5.08

5.07

5.03

4.96

4.96

4.95

4.87

4.86

4.86

4.81

4.81

4.80

4.79

4.76

4.75

4.68

4.65

4.56

4.53

4.51

4.49

4.48

4.48

4.48

4.42

4.41

4.30

4.30

4.19

4.14

4.10

4.07

4.03

4.02

4.02

3.91

3.87

3.87

3.86

3.82

3.71

3.50

Is an unemotional person; tends not to experience strong emotions (low placement implies a highly emotional person).

Denies the presence of anxiety and conflicts; tends to convince himself/herself that unpleasant thoughts and feelings do not exist; deceives self into thinking everything is fine, when everything is not fine. 
Table A2 continued

55 Is self-defeating; acts in ways that frustrate, hurt, or undermine own chances to get what he/she wants.

Tends to blame others for own mistakes, failures, and shortcomings.

Is condescending toward others; acts superior to others.

Is self-dramatizing; theatrical; prone to exaggerate feelings; seeks attention.

Has hostility toward others (whether or not the hostile feelings are actually expressed). 3.24

$\begin{array}{ll}\text { Feels cheated and victimized by life; self-pitying; feels sorry for self. } & 2.85\end{array}$

Is guileful, deceitful, manipulative, opportunistic; takes advantage of others. $\quad 2.70$

$\begin{array}{ll}36 & \text { Tends to undermine, obstruct, or sabotage other people. } \\ 36\end{array}$

Note: Countries include: Canada, China, Czech Republic, France, Poland, Russia, Singapore, Slovakia, South Africa, South Korea, Switzerland, UK, US. 